\title{
Guided Walking to Direct Pedestrians toward the Same Destination
}

\author{
Nobuhito Sakamoto \\ Osaka University \\ Suita, Osaka, Japan \\ nobuhito-sakamoto@hiel.ist.osaka-u.ac.jp
Masataka Kurokawa
Osaka University \\ Suita, Osaka, Japan \\ masataka-kurokawa@hiel.ist.osaka-u.ac.jp
}

\author{
Masahiro Furukawa \\ Osaka University \\ JST PRESTO \\ CiNet \\ Suita, Osaka, Japan \\ m.furukawa@ist.osaka-u.ac.jp
}
Taro Maeda
Osaka University
CiNet

Suita, Osaka, Japan

t_maeda@ist.osaka-u.ac.jp
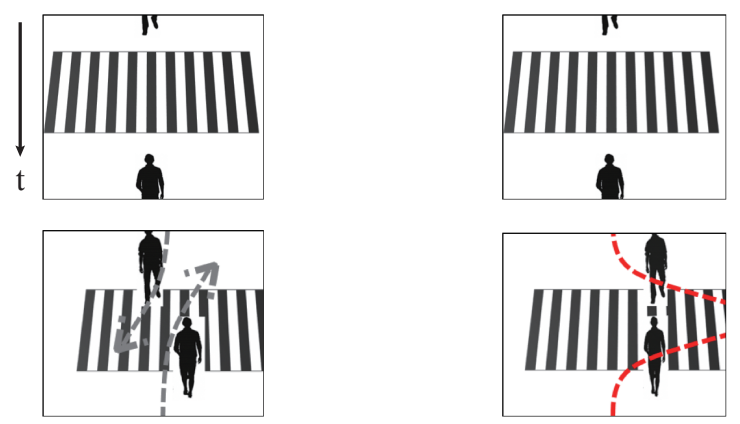

guidance sheet such that a pedestrian travelling in any direction can be guided toward a given point. In experiments, our system successfully guided a pedestrian along the same direction regardless of the direction of travel using the walking guidance sheet. The induction effect of the proposed method was also evaluated.

\section{CCS CONCEPTS}

- Human-centered computing $\rightarrow$ User centered design; Interaction design theory, concepts and paradigms; Mixed / augmented reality; Ubiquitous computing.

\section{KEYWORDS}

vection, visual stimulus, walking guidance, pedestrian

\section{ACM Reference Format:}

Nobuhito Sakamoto, Masahiro Furukawa, Masataka Kurokawa, and Taro Maeda. 2019. Guided Walking to Direct Pedestrians toward the Same Destination. In Augmented Human International Conference 2019 (AH2019), March 11-12, 2019, Reims, France. ACM, New York, NY, USA, 8 pages. https://doi.org/10.1145/3311823.3311835

\section{INTRODUCTION}

Pedestrians are guided in everyday life through signs or arrows indicating direction, or by vocal instructions. This information requires such processes as the presentation of information, acquisition of pedestrian-related information and its interpretation, and gauging pedestrian behavior based on this. It takes time for the pedestrian to act once the relevant information is presented. Therefore, we

Permission to make digital or hard copies of part or all of this work for personal or classroom use is granted without fee provided that copies are not made or distributed for profit or commercial advantage and that copies bear this notice and the full citation on the first page. Copyrights for third-party components of this work must be honored

For all other uses, contact the owner/author(s).

AH2019, March 11-12, 2019, Reims, France

(C) 2019 Copyright held by the owner/author(s)

ACM ISBN 978-1-4503-6547-5/19/03.

https://doi.org/10.1145/3311823.3311835

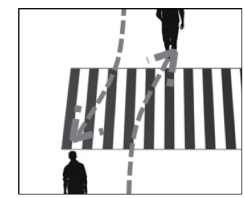

Conventional Method
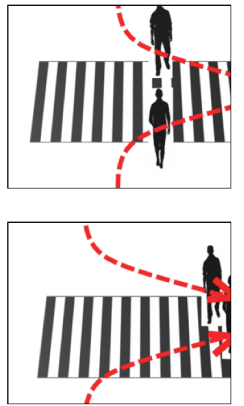

Proposed Method
Figure 1: Induction Diagram of Conventional and Proposed Method

propose here a direct guidance method that does not require interpretation. This method can help efficient queuing and prevent falls in areas with large crowds, such as public transportation centers. As people use visual cues to stabilize posture when walking, a guidance method that provides pedestrians with a visual stimulus is effective. Therefore, we focus on visually induced self-motion sensation (vection), and present a visual stimulus to the environment that causes the postural reflex to implement guidance when walking. In previous work [5, 31], a lenticular lens was used as optical element, and walking along any given direction was not dependent on the general direction of travel. It was demonstrated that guidance can be used for collision avoidance.

By applying the methods proposed in this work, we devise and implement a method that provides an induction effect depending on the direction of walking to guide a pedestrian coming from any direction to a given point. This induction effect depends on 
the direction of the pedestrian, unlike in the conventional method where it does not depend on this direction and generates the same effect in one-dimensional direction shown in Fig.1. This makes it possible to provide a gradient to the induction effect along a 2D direction. As a first step of induction toward to one point, we propose a guiding method toward the same direction shown in Fig.1.

\section{RELATED WORKS}

\subsection{Guided Walking using Sensory Illusion}

Currently, it is very common that pedestrians use smartphones for navigation [2]. Given that they are easier to hold, it is reasonable to think that pedestrians would prefer a smartphone to a map for guided navigation [1]. We examine here the creation of an "illusion" whereby users feel as if their hands are held as they are guided. Illusion has long been noted as a means for achieving an experience that cannot otherwise be realized or is difficult to realize.

Many methods [28] have been proposed, such as a belt where the part closest to the destination vibrates on the waist to continually present the direction of the destination while walking. Although this is one method of guided walking using a portable device, it is not an illusion in the sense used here, as the pedestrian is assumed to continue walking while conscious of the vibrating part of the belt. A method where the pedestrian is notified of the given position by sounds instead of vibrations has also been proposed [25]. Methods that rely on sensations on the human skin for guided direction include gyroscopic effects [18] and skin pulling [6]. They also present the bearing "as a sign" in the same way. In the above research [1] however, the illusion of a continuous traction force was implemented by using the fact that the human tactile sense is nonlinear. This provides the experience of being guided as if one's hand were being pulled, without making the user symbolically aware of the azimuth of the destination.

The guided walking method that directly drives the body of the pedestrian does not require symbolic interpretation by the user. Methods that do not use sensory illusion include directly stimulating the muscles of the lower limb [22], and reproducing the state of walking virtual ruts by tilting the sole [4]. On the contrary, the use of sensory illusions has the advantage that there is room for pedestrians to maintain the safety of the guidance. One example is that of a method of creating the illusion of a sense of equilibrium (vestibular sensation) using a weak current $[14,15]$. It is sufficiently effective for the body to respond arbitrarily without the pedestrians being conscious of this. A method where the outer ear experiences a pulling sensation [12] has also been proposed as one that has an effect close to the guiding effect generated by the illusion of equilibrium.

The concept of optical illusion [17] shows that while it is possible to transmit the direction of the destination without depending on symbols, which can provide a pedestrian with an impression of movement by observing it in the peripheral visual field, this is not direct guiding without the pedestrian being conscious of it. A method has been proposed [16] to induce the illusion of walking where the pedestrians do not notice the direction. The essence of this method is to continuously give the impression that the person is walking in a straight line, where in fact this direction is slightly bent. When comparing the optical illusion with visual guidance, note that the preconditions are different. The former assumes that the pedestrian is stationary and the latter assumes that he or she is walking. The illusion used in guided walking described in this research falls in the former class at first glance, but essentially is in the latter.

\subsection{Visual Stimulation and Vection}

Lishman et al. [13] conducted experiments that showed inconsistency between the somatosensory sensation and vision, and concluded that the latter is more dominant in controlling the body. Therefore, we focus on the visual, inductive sensation of motion (vection) for guided walking that does not require the attachment / detachment of a device.

Vection (visual inductive self-kinesthetic sensation) is an illusion whereby the observer perceives a visual stimulus as moving in the direction opposite to its actual direction. We arrange the effect of vection at the azimuth angle in the field of view. According to Blandt et al., the larger the area of stimulation, the stronger the induction of vection [3]. It was also subsequently reported that peripheral vision strongly attracts vection $[8,19,23]$. For example, Johansson [11] showed that the visual stimulus in peripheral vision is more effective than in central vision in case of vection. Hatada et al. [8] analyzed the functional difference between the central vision and the peripheral visual field regions using the inclination of the body provided by a visual stimulus as an index, and concluded that the central part of the retina is sensitive to slow motion and its periphery is highly sensitive to rapid motion. Hayahuku [9] showed that the reaction time in objective motion is longer in peripheral vision than in central vision, whereas Yamagishi et al. [30] compared peripheral vision with central vision. According to Ito et al., experiments where diffusion and convergence stimuli were superimposed on planes of different depths have shown that exercising the physical stimulus stimulates the domination of vection [10].

We now consider the characteristics of the motion stimulus. With regard to spatial frequency, rough stripes (low spatial frequency) have been shown to induce vection more efficiently than fine stripes [24]. Another study has shown an interaction between the roughness of the stripes and the position of the presentation field of view, and concluded that rougher stripes are better in the peripheral visual field whereas finer ones are more effective in the central visual field) [20]. According to a study by Kure et al. [29], the spatial frequency of the visual stimulus ranges from 0.037 to 0.057 [cycle/deg], which is the range that induces the most vection. Regarding visual speed, there is a linear correspondence between the self-motion speed perceived as the stimulation speed up to 100 [deg/s] (increasing stimulation speed, the sense of movement speed of itself also rises correspondingly, and the contribution becomes smaller at higher speeds) [7].

By combining these, we can conclude that vection can be induced when the visual stimulus moves behind the scene and the surroundings, spatial frequency is low, and the area is large, that is, the part perceived as the "ground" does not conventionally move.

With reference to the above parameters, in Experiment 1 of this study, we verified two parameters, spatial frequency and velocity, effective for inducing walking toward a point. 
Further, in a study using vection as a means of inducing walking $[5,31]$, Yoshikawa et al. obtained a dynamic visual stimulation without a power supply and focused on an optical element known as a lenticular lens for visual presentation suitable for environmental settings. They proposed the "vection field," where walking is induced by using the lenticular lens for visual stimulus effective for guided walking over a wide range (Fig.1). In this method, pedestrians are guided by a visual stimulus from the vection field moving vertically to the right with respect to the direction of motion of the pedestrian. By applying this, we devise a method to provide the induction effect depending on the direction of motion (Fig.1) to realize the effect induced on a pedestrian coming from any direction toward one point. This method was verified in Experiment 2.

\section{PROPOSED METHOD}

\subsection{Visual Motion with Parallax Barrier}

To realize guidance toward a point, it is necessary to invert the induced polarity depending on the perspective taken, instead of guiding in a uniform direction independent of it.

Although the lenticular lenses adopted in conventional methods $[5,31]$ can produce dynamic visual stimuli completely a without power supply, there are restrictions on the number of realizable symbols due to the lens standard and the resolution of the printer. Therefore, we focus on a phenomenon called the slit view. Slit viewing refers to a phenomenon that gives the impression that the entire shape is visible when observing a figure through an elongated slit [21], despite the fact that the figures are presented sequentially and fragmentally across the slits. Using this phenomenon, a stereoscopic video presentation of horizontal 360 degrees was implemented $[27,32]$ in a cylindrical booth-type display to be seen with the naked eye.

In this method, acrylic lenses with patterns printed on the front and back surfaces of a transparent acrylic plate were used as optical elements. By providing the barrier layer (Fig.2) on the surface of the acrylic lens, it is possible to freely select the pattern layer (Side View in Fig.2) to be exposed with respect to the viewpoint taken By changing the thickness $h[\mathrm{~mm}]$ of the acrylic plate, the rate of flow can be controlled. By printing a pattern layer (Top View in Fig.2), in which black lines with width $l[\mathrm{~mm}]$ and length $L[\mathrm{~mm}]$ are drawn alternately with inclinations $\alpha$ [deg] and - $\alpha$ [deg] on the back of the acrylic plate, and are filled at even intervals (fill width $D[\mathrm{~mm}]$ with white at an interval $d[\mathrm{~mm}])$ ), with the barrier layer (Top View in Fig.2) on the surface of the acrylic plate, black lines of the pattern layer can be exposed through the acrylic plate within the unfilled width $d$ in the barrier layer, and an effect like that produced by slits can be achieved. As a human being moves on this acrylic lens, the black lines move and are exposed (Fig.3). Moreover, by designing the system so that

$$
L \sin \alpha[m m]+\frac{l}{\cos \alpha}[m m]=\frac{D+d}{2}[m m]
$$

is satisfied, the induced polarity can be reversed depending on the viewpoint, as shown in Fig.2, where the symbol of range A' appears when viewed from range $A$, and the symbol of range $B$ ' appears when viewed from range $B$.

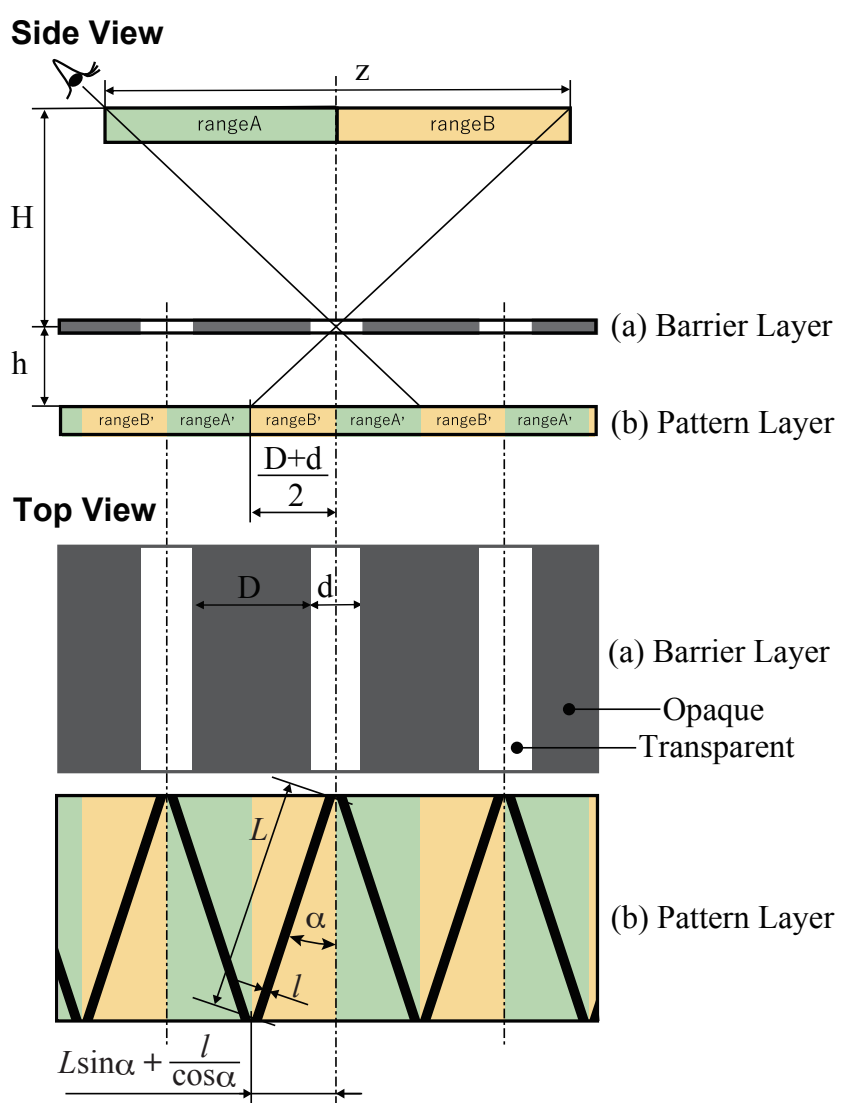

Figure 2: Relationship between Acrylic Sheet and Viewpoint

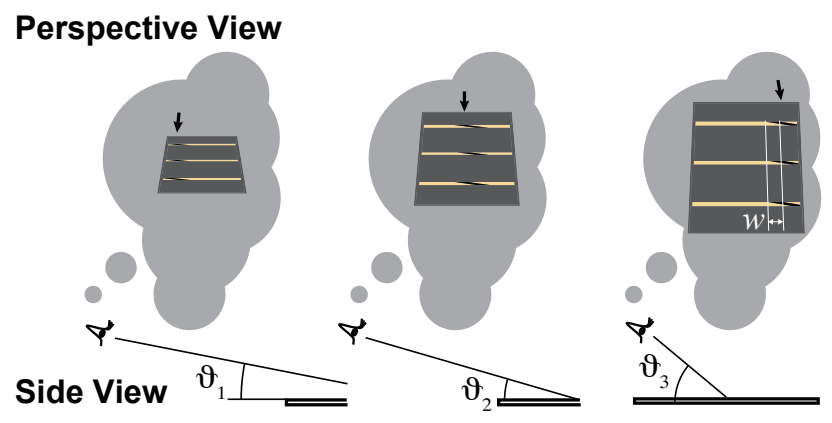

Figure 3: Expression Pattern by View Point and View Angle

\subsection{Visual Stimulus}

As experimental stimuli, black and white stripes were used in the same way as in a few previous studies [5, 31], and their velocities/spatial frequencies were used as variable parameters. As mentioned in Section 2.2, the range of the spatial frequency of the visual stimulus was set in the range $0.037-0.057$ [cycle/deg], which induces the most vection [29]. As visual speed contributes little to self-perceived speed at stimulus speeds above 100 [deg/s] [7], the spatial frequencies were set to $0.02,0.05,0.08,0.11$, and 0.14 [cycle/deg], and the stimulation rate was set to $20,40,60,80$, and 
$100[\mathrm{deg} / \mathrm{s}]$. Twenty-six measurements were repeated for each of a total of 26 conditions, i.e., 25 conditions based on a combination of spatial frequency and stimulation rate plus control conditions without stimulation. The order of the stimuli order was completely random. The subjects were 10 men aged 22 to 24 years old. All subjects had normal visual acuity or normal corrected vision.

\subsection{Experimental Environment}

As HMD (head-mounted display), we used the HTC Vive Pro (resolution: $1440 \times 1600$ [pixel] per eye, total $2880 \times 1600$ [pixel], viewing angle: 110 [deg]). Virtual environment is developed on Unity, and the light at the HMD was (X: 0, Y: 3, Z: 0), Rotation (X: 50, Y: 30, Z: 0 ), Intensity: 1 in the Unity coordination system. The visual stimulus was presented in the virtual environment within the HMD $3[\mathrm{~m}]$ from the position of $2[\mathrm{~m}]$ in a straight line from the starting position of guided walking with a range of 2.5 meters on both sides $\left(3 \times 5=15\left[\mathrm{~m}^{2}\right]\right)$ as shown in Fig.4. A red line was drawn $4.7[\mathrm{~m}]$ from the start. The subject was instructed to stop walking when the line was reached. Subjects were also instructed to walk while watching the red line. The position of the HMD during walking was tracked (90 [fps]) and used as head position data during walking. Moreover, each spatial frequency/stimulus rate was set for each subject so that both were representative when the cylindrical surface came into contact with the floor when looking directly under the upright state.

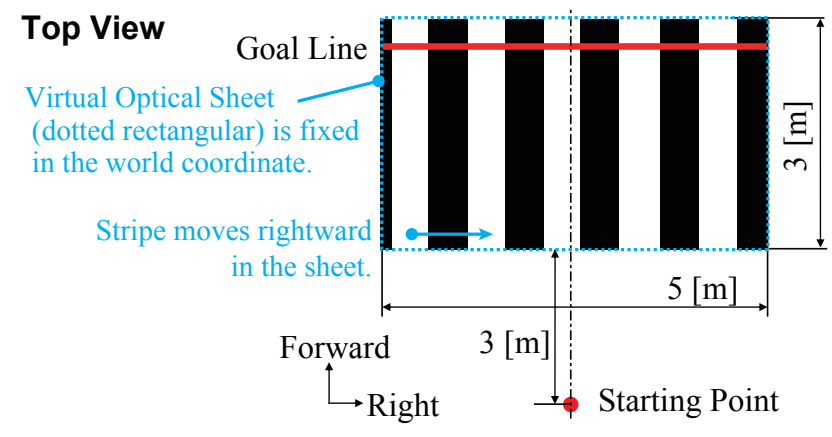

Figure 4: Experimental Condition with No Barrier Layer Condition

\subsection{Results}

In the experiment, the difference between the initial and end positions of the HMD (perpendicular to the direction of travel) was used as an index of the inductive effect (hereinafter referred to as "horizontal difference"). The visual stimulus flowed from left to right in the direction of travel, and the HMD was positive along the right. By comparing the control conditions and those with the visual stimulus for each subject, and applying the test among conditions for the subject using the Holm method, significant differences were found in eight out of 10 subjects. However, one of the eight subjects showed an effect different from the assumed induction effect (the subject walked straight when there was a visual stimulus, and in a curve under the control conditions). Thus, seven of 10 subjects were grouped as having been affected and three as not having been affected.
Fig. 5 shows the inter-subject mean shift in horizontal axis for each of the seven subjects by spatial frequency. We conducted oneway ANOVA under the spatial frequency factor [26]. Main effect of spatial frequency factor is significant $(F(5,30)=17.61, p<.01)$. As a result of multiple comparisons using the Holm method, a significant difference was found between the control conditions and each stimulus condition $(p<.05)$. The control condition is significantly lower than the other conditions. It was revealed that guided walking is realized under the spatial frequency condition between from 0.02 to 0.14 [cycle/deg].

Fig. 6 shows the inter-subject mean shift in horizontal axis for each of the same seven subjects by angular velocity. Main effect of angular frequency factor is significant $(F(5,30)=13.84, p<.01)$. Multiple comparisons using the Holm method showed a significant difference was found between the control conditions and each stimulus condition $(p<.05)$. The control condition is significantly lower than the other conditions. Control condition does not provide stripe motion towards the right side on the floor. So it is found that the stripe motion induce horizontal shift significantly. In contrast, the inter-angular velocity condition does not provide significant condition. Thus, constantly the guided walking effect is obtained under the angular velocity above 20 [deg/s].

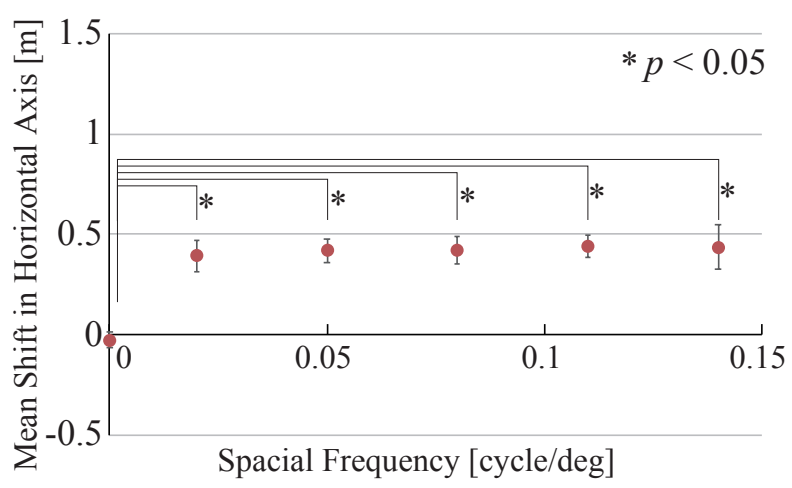

Figure 5: Mean Shift by Spatial Frequency Among Subjects Responded Positively ( $\mathrm{N}=7$ in 10)

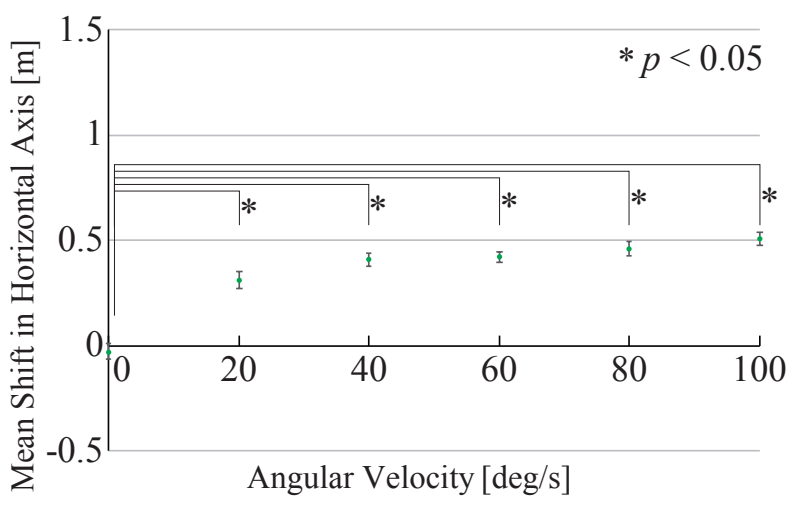

Figure 6: Mean Shift for Angular Velocity by Subject Responded Positively ( $\mathrm{N}=7$ in 10$)$ 


\subsection{Discussion}

The experimental results show that it is possible to induce walking by providing a visual stimulus of black and white stripes flowing in one direction on the floor. To summarize and compare the positive and negative result $(\mathrm{N}=10)$, we set the spatial frequency multiplied by the visual stimulus (the number of cycles for which the stimulus moves per second [cycle/s] as a new integrated unit (hereinafter "cycle velocity"), plotted for each condition shown in Fig.7. The horizontal axis shows the passing number of one stripe cycle per second. The vertical axis shows the mean shift of walking in horizontal axis. The red plots indicate positive results $(\mathrm{N}=7)$ and black open circle plots indicate negative results $(\mathrm{N}=3)$. The error bar shows standard deviation.

These plot seems to have a saturation value in terms of cycle velocity. So we delivered an approximate curve was obtained by the modified cumulative normal distribution function using leastsquare method. Assuming that the obtained results are delivered point-symmetrically, we additionally duplicate the sampled data and rotated them around the origin point. Then, the modified cumulative normal distribution function is fitted to the twice number of the sampled data. The green curve is obtained from the positive responses, and the brue curve is obtained from the negative responses.

The induction effect converged as cycle velocity increased. Gradient values around the origin point of Fig. 7 are 0.735 of positive responses and 0.192 of negative responses. These values mean that where the induced guided walking effect linearly increases higher when the cycle velocity is from 0 to approximately 1 [cycle/s]. The gradient of the positive responses is much higher than the one of the negative responses. Above cycle velocity 1 [cycle/s], the effect seems to be saturated. Then, the asymptic line shows the two saturation value as asymptic value from the fitting curve: 0.585 [m] of positive responses and $0.093[\mathrm{~m}]$ of negative responses. When it exceeded 3.0 [cycle/s], it reached $95 \%$ of the convergence value. In other words, if a visual stimulus satisfying at least 3.0 [cycle/s] is provided, guided walking can be steadily induced.

\section{EFFECT OF BARRIER}

\subsection{Visual Stimulus}

Black and white stripes were used as experimental stimuli in the same way as in the experiment in Section 3, where the cycle velocity was within the monotonically increasing section of the asymptic value in Fig.7, i.e., 1.0 [cycle/s] (spatial frequency $=0.05$ [cycle $/ \mathrm{deg}]$, stimulus rate $=20[\mathrm{deg} / \mathrm{s}]$ ), and was located in the section of $95 \%$ or more of the asymptotic value of curve 4.0 [cycle/s] (spatial frequency $=0.05[\mathrm{cycle} / \mathrm{deg}]$, stimulus rate $=80[\mathrm{deg} / \mathrm{s}])$. The widths of the barrier were set to $0.05,0.10$, and 0.15 [m], and the barrier intervals were $0.05,0.10$, and $0.15[\mathrm{~m}]$. As a comparison condition of these combinations 2 conditions $\times 3$ conditions $\times 3$ conditions $=$ 18 conditions, no barrier 2 conditions (cycle velocity $=1.0$ [cycle s], 4.0 [cycle s] )plus no stimulus 1 condition, total 21 conditions were measured three times. The order of the stimuli was completely random. The subjects were seven males aged 22 to 24 who had been influenced by the stimuli in the above experiment. All subjects had normal visual acuity or normal corrected vision.

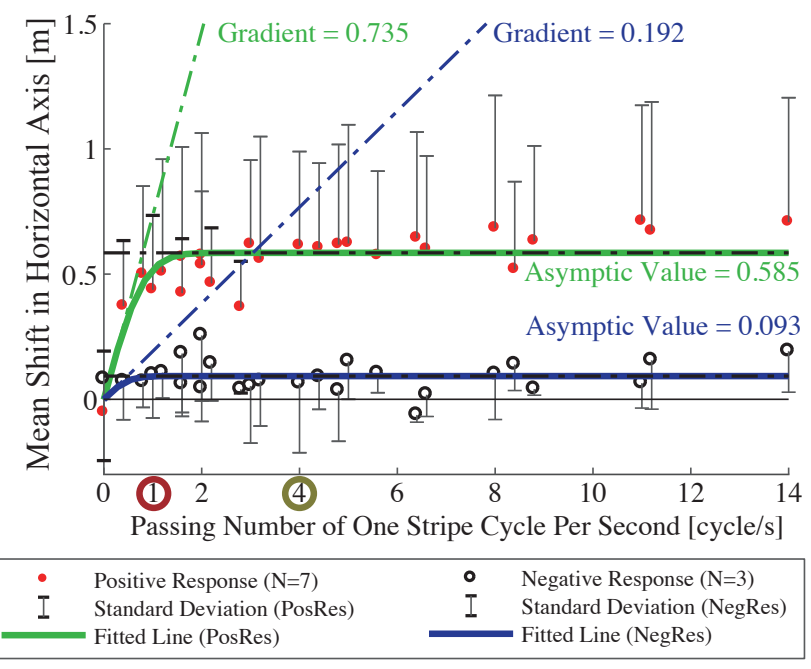

Figure 7: Mean Shift per Cycle Velocity by Subject

\subsection{Experimental Environment}

As HMD, we used the HTC Vive Pro (resolution: 1440 x 1600 [pixel] per eye, total $2880 \times 1600$ [pixel], viewing angle: 110 [deg]. Virtual environment is developed on Unity as same as subsection 3.3, and the light in the HMD was at (X: 0, Y: 3, Z: 0), Rotation (X: 50, Y: 30, Z: 0), Intensity: 1 in the Unity coordination system. The visual stimulus was presented in the virtual environment within the HMD $3[\mathrm{~m}]$ from the straight-line direction of motion of the subject from the start position, with a range of $2.5 \mathrm{~m}$ on both sides $\left(3 \times 5=15\left[\mathrm{~m}^{2}\right]\right.$ (Fig.8). A red line was drawn 4.7 [m] from the start position, and subjects were instructed to stop walking when the line was reached. They were also instructed to walk while watching the red line. The position of the HMD during walking was tracked ( 90 [fps]) and used as head position data during walking. Spatial frequency/stimulus rate was set for each subject so that the spatial frequency and the stimulus rate at the tangential plane, where the cylindrical surface came into contact with the floor, were representative values when looking directly under the upright state.

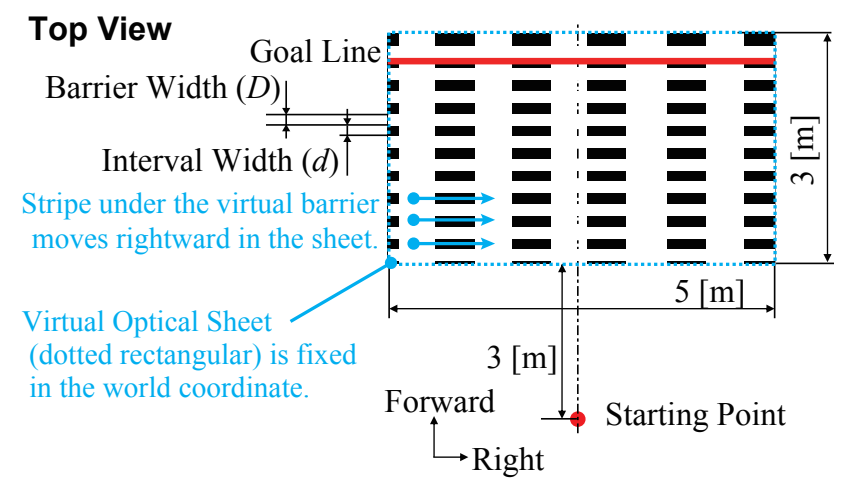

Figure 8: Experimental Environment 


\subsection{Results}

In this experiment, the difference between the initial and end positions of the HMD (perpendicular to the direction of motion) was used as an index of the induction effect (horizontal difference) as in the experiment in Section 3. The visual stimulus flowed from left to right along the direction of travel, and the position of the HMD was positive to the right. By comparing the control condition and the visual stimulus for each subject, and testing the conditions, we added the two data sets obtained from the control condition as well as the no barrier condition to the obtained data. The result of one-way ANOVA under each experimental condition in order to compare the control condition obtained by the previous experiment.

Fig.9 shows the inter-subject mean shift in horizontal axis for each of the seven subjects at a certain barrier width. One parent population includes five conditions: one control condition, no barrier condition, and three barrier width $D[\mathrm{~m}]$ condition under each cycle velocity condition 1 or 4 [cycle/s]. Each data represents mean value of three times trial under the each same condition. We conduct inter-subject two-way ANOVA. The main effect of cycle velocity factor is marginally significant $(F(1,6)=4.68, p<.01)$, and main effect of barrier width is significant $(F(4,24)=16.46, p<.01)$. Interaction between the cycle velocity factor and barrier width is significant $(F(4,24)=3.21, p<.05)$. Multiple comparisons of using the Holm method showed a significant difference was found between the control and each stimulus condition $(p<.05)$. The control condition is significantly lower compared with the other condition. There is no statistical significance when between no barrier condition and other three barrier width conditions of more than $0.05[\mathrm{~m}]$.

Fig.10 shows the inter-subject mean shift in horizontal axis for each of the same subjects at different interval width. The parent population includes five conditions: one control condition, no barrier condition, and three barrier interval $d[\mathrm{~m}]$ condition under each cycle velocity condition 1 or 4 [cycle/s]. Each data represents mean value of three times trial under the each same condition. We conduct inter-subject two-way ANOVA. The main effect of cycle velocity factor is marginally significant $(F(1,6)=4.68, p<.01)$, and main effect of barrier width is significant $(F(4,24)=16.41, p<.01)$. Interaction between the cycle velocity factor and barrier interval is significant $(F(4,24)=2.80, p<.05)$. Multiple comparisons of using the Holm method showed a significant difference was found between the control and each stimulus condition $(p<.05)$. The control condition is significantly lower compared with the other condition. As mentioned in Fig.9, there is no statistical significance when between no barrier condition and other three barrier interval conditions of more than $0.05[\mathrm{~m}]$.

\subsection{Discussion}

The experimental results show that walking can be induced by adding a barrier layer to the visual stimulus of a black and white stripe flowing in one direction on the floor surface. Using barrier width $D[\mathrm{~mm}]$ and barrier interval $d[\mathrm{~mm}]$ as mentioned in subsection 3.1, the ratio of the barrier [\%] to the area of the total visual stimulus was set as a new integrated unit (hereinafter "barrier ratio"). We define the unit as follows: barrier ratio $=\frac{d}{d+D}[\%]$

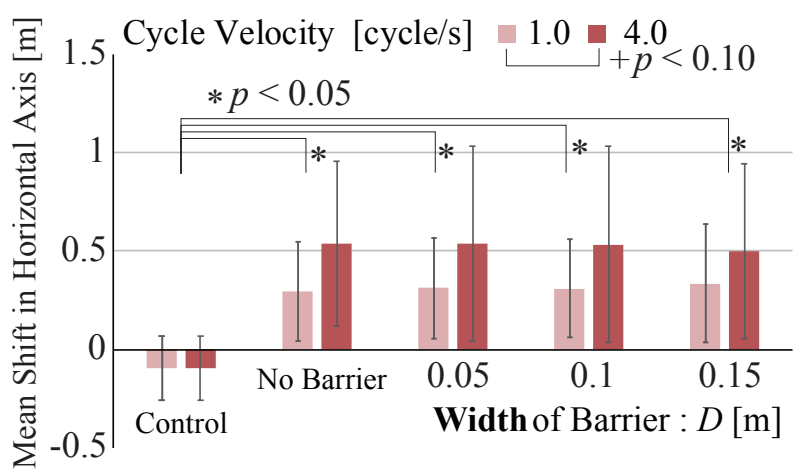

Figure 9: Mean Shift for Each Barrier Width by Subject

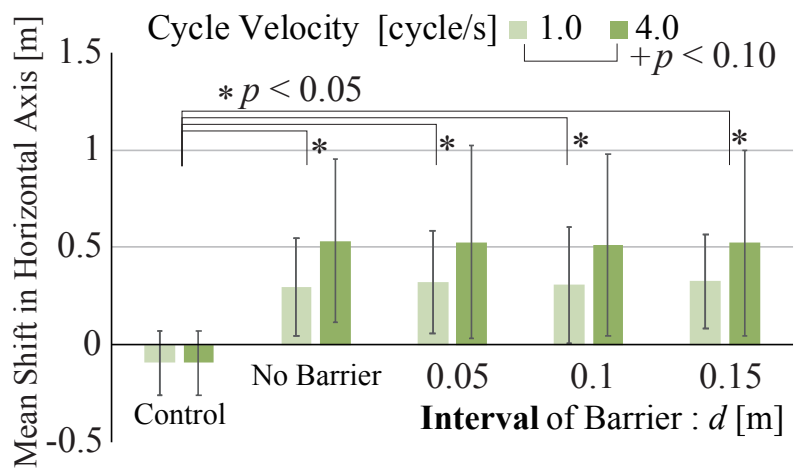

Figure 10: Mean Shift per Barrier Interval by Subject

Plotted for each condition shown in Fig.11 using the unit. The horizontal axis show the barrier ratio [\%]. The vertical axis shows the mean shift in horizontal axis[m]. The error bar indicates standard deviation. It was found that the induction effect converged as barrier ratio increased. Approximate curves is obtained by the modified cumulative normal distribution function using least-square mentioned in subsection 3.5. Yellow and red curve are obtained when cycle velocity is 4 and 1 [cycle/s]. The asymptic line shows the two saturation value as asymptic value from these fitting curve: 0.526 and $0.316[\mathrm{~m}]$ when cycle velocity is 4 and 1 [cycle/s].

Above barrier ratio 25[\%], the induction effect seems to be saturated. Barrier ratio 25[\%] means the barrier does not cover a space of one-fourth all surface. Though this narrow interval, the saturated value $0.526[\mathrm{~m}]$ under the 4 [cycle/s] is very close to 0.585 [m] of positive responses shown in Fig.7 with open yellow circle. It is revealed that the experimental result shows that the barrier ratio 25[\%] does not harm the induction effect. Interestingly, the same number of barrier ratio remains the induction effect under the cycle velocity 1 [cycle/s]. In contrast, the asymptic value decreases to 0.316 . The induction effect is on the gradient line near the cycle velocity 1 [cycle/s] condition shown in Fig.7 with open red circle on the horizontal axis. The induction effect reached $95 \%$ of the convergence value when the barrier ratio was 25 [\%]. In other words, walking can be steadily induced if a visual stimulus with at least a barrier ratio of 25 [\%] is given. 


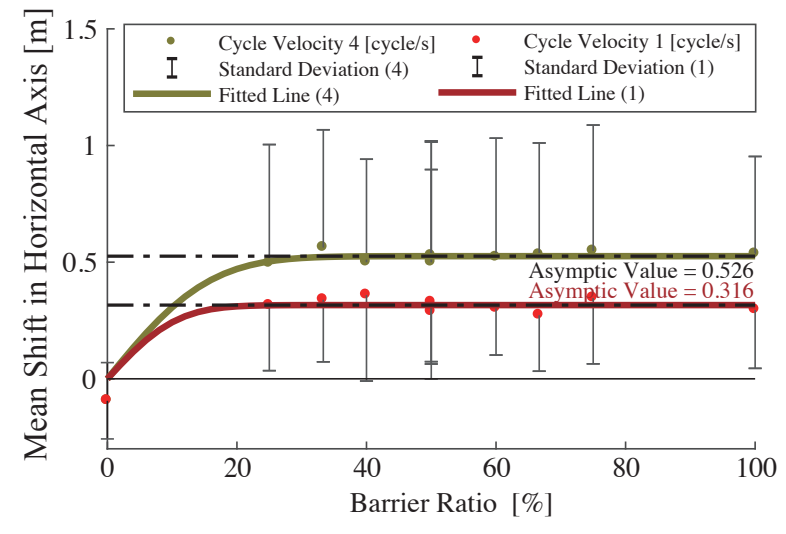

Figure 11: Average horizontal difference per barrier ratio by subject

\section{CONCLUSION}

In this paper, we propose a guided walking method using visual motion appeared on the floor to induce self-motion illusion (vection). Novelty of this proposal is to switch guiding direction depending on the user's view direction. In contrast, the previous method with the use of lenticular lens does not have wide viewing angle enough to realize the purpose mentioned before. So we propose the parallax barrier method to make the viewing angle wide enough as well as to reverse the direction of visual motion from the view point at the opposite side. Therefore, the proposed method can invert the polarity of the direction of the moving stimulus.

To evaluate the proposed method, we conducted two experiments in the virtual world. Firstly, we created a new integrated unit "cycle velocity", which works as an evaluation function leading to the induction effect. Secondly, we evaluated the effect of several conditions in terms of index "cycle velocity" derived from the spatial frequency of the visual stimulus and the stimulation velocity. Lastly, the interaction between barrier and visual motion is evaluated.

Result shows that a constant walking induction effect can be obtained when the cycle velocity is 3.0 [cycle/s] or higher. Similar walking induction effect is obtained when the index (barrier ratio) derived from barrier width and ratio is $25(\%)$ or more. We will apply these findings to the actual multi-layered optical sheet design.

\section{ACKNOWLEDGMENTS}

This research was supported by JST, PRESTO Grant Number JPMJPR15D7, Japan.

\section{REFERENCES}

[1] Tomohiro Amemiya, Hideyuki Ando, and Taro Maeda. 2008. Lead-me Interface for a Pulling Sensation from Hand-held Devices. ACM Trans. Appl. Percept. 5, 3 , Article 15 (Sept. 2008), 17 pages. https://doi.org/10.1145/1402236.1402239

[2] Masatoshi Arikawa, Shin'ichi Konomi, and Keisuke Ohnishi. 2007. Navitime Supporting Pedestrian Navigation in the Real World. IEEE Pervasive Computing 6, 3 (July 2007), 21-29. https://doi.org/10.1109/MPRV.2007.61

[3] Th. Brandt, J. Dichgans, and E. Koenig. 1973. Differential effects of central versus peripheral vision on egocentric and exocentric motion perception. Experimental Brain Research 16, 5 (01 Mar 1973), 476-491. https://doi.org/10.1007/BF00234474

[4] Martin Frey. 2007. CabBoots: Shoes with Integrated Guidance System. In Proceed ings of the 1st International Conference on Tangible and Embedded Interaction (TEI
'07). ACM, New York, NY, USA, 245-246. https://doi.org/10.1145/1226969.1227019

[5] Masahiro Furukawa, Hiromi Yoshikawa, Taku Hachisu, Shogo Fukushima, and Hiroyuki Kajimoto. 2011. "Vection Field" for Pedestrian Traffic Control. In Proceedings of the 2Nd Augmented Human International Conference (AH '11). ACM, New York, NY, USA, Article 19, 8 pages. https://doi.org/10.1145/1959826.1959845

[6] B. T. Gleeson, S. K. Horschel, and W. R. Provancher. 2009. Communication of direction through lateral skin stretch at the fingertip. In World Haptics 2009 Third foint EuroHaptics conference and Symposium on Haptic Interfaces for Virtual Environment and Teleoperator Systems. 172-177. https://doi.org/10.1109/WHC. 2009.4810804

[7] Bernd De Graaf, Alexander H. Wertheim, Willem Bles, and Jan Kremers. 1990. Angular velocity, not temporal frequency determines circular vection. Vision Research 30, 4 (1990), 637 - 646. https://doi.org/10.1016/0042-6989(90)90074-U

[8] Toyohiko Hatada, Haruo Sakata, and Hideo Kusaka. 1979. Induced Effect of Direction Sensation and Display Size. The Journal of the Institute of Television Engineers of Japan 33, 5 (1979), 407-413. https://doi.org/10.3169/itej1978.33.407

[9] Sumiko Hayafuku. 1969. Study on Perception Characteristics in Peripheral VisionRelationship Stimulation Emergence Azimuth and Reaction Time. Bulletin of Industrial Arts Institute 58 (1969), 11-16.

[10] Hiroyuki Ito and Ikuko Shibata. 2005. Self-motion perception from expanding and contracting optical flows overlapped with binocular disparity. Vision Research 45 , 4 (2005), 397 - 402. https://doi.org/10.1016/j.visres.2004.11.009

[11] Gunnar Johansson. 1977. Studies on Visual Perception of Locomotion. Perception 6, 4 (1977), 365-376. https://doi.org/10.1068/p060365 arXiv:https://doi.org/10.1068/p060365 PMID: 917725.

[12] Yuichiro Kojima, Yuki Hashimoto, Shogo Fukushima, and Hiroyuki Kajimoto. 2009. Pull-navi: A Novel Tactile Navigation Interface by Pulling the Ears. In ACM SIGGRAPH 2009 Emerging Technologies (SIGGRAPH '09). ACM, New York, NY, USA, Article 19, 1 pages. https://doi.org/10.1145/1597956.1597975

[13] J R Lishman and D N Lee. 1973. The Autonomy of Visual Kinaesthesis. Perception 2, 3 (1973), 287-294. https://doi.org/10.1068/p020287 arXiv:https://doi.org/10.1068/p020287 PMID: 4546578.

[14] T. Maeda, H. Ando, T. Amemiya, N. Nagaya, M. Sugimoto, and M. Inami. 2005. Shaking the World: Galvanic Vestibular Stimulation As a Novel Sensation Interface. In ACM SIGGRAPH 2005 Emerging Technologies (SIGGRAPH '05). ACM, New York, NY, USA, Article 17. https://doi.org/10.1145/1187297.1187315

[15] T. Maeda, H. Ando, and M. Sugimoto. 2005. Virtual acceleration with galvanic vestibular stimulation in a virtual reality environment. In IEEE Proceedings. VR 2005. Virtual Reality, 2005. 289-290. https://doi.org/10.1109/VR.2005.1492799

[16] Keigo Matsumoto, Yuki Ban, Takuji Narumi, Yohei Yanase, Tomohiro Tanikawa, and Michitaka Hirose. 2016. Unlimited Corridor: Redirected Walking Techniques Using Visuo Haptic Interaction. In ACM SIGGRAPH 2016 Emerging Technologies (SIGGRAPH '16). ACM, New York, NY, USA, Article 20, 2 pages. https://doi.org/ $10.1145 / 2929464.2929482$

[17] Ikuya Murakami. 2008. Illusion sheet of pedestrian passage. Patent No. JP4159518B2, Filed Jun. 17th., 2004, Issued Oct. 25th., 2008.

[18] Norio Nakamura. 2003. Development of Human Navigation System "HapticNavi" using GyroCube. 15th Triennial Congress of the International Ergonomics Association 2003 (2003). https://ci.nii.ac.jp/naid/10020197325/en/

[19] Hitoshi Ohnishi, Kaname Mochizuki, and Yuji Sugimoto. 2003. Measuring the Reality Given by Highly Realistic Surrounding Display Systems : Application of an ARX Model to Observer's Body-sway Data. The Transactions of the Institute of Electronics,Information and Communication Engineers B 86, 1 (jan 2003), 45-56. https://ci.nii.ac.jp/naid/110003170326/en/

[20] Stephen Palmisano and Barbara Gillam. 1998. Stimulus Eccentricity and Spatial Frequency Interact to Determine Circular Vection. Perception 27, 9 (1998), 10671077. https://doi.org/10.1068/p271067 arXiv:https://doi.org/10.1068/p271067 PMID: 10341936.

[21] Theodore E. Parks. 1965. Post-Retinal Visual Storage. The American fournal of Psychology 78, 1 (1965), 145-147. http://www.jstor.org/stable/1421101

[22] Max Pfeiffer, Tim Dünte, Stefan Schneegass, Florian Alt, and Michael Rohs. 2015. Cruise Control for Pedestrians: Controlling Walking Direction Using Electrical Muscle Stimulation. In Proceedings of the 33rd Annual ACM Conference on Human Factors in Computing Systems (CHI '15). ACM, New York, NY, USA, 2505-2514. https://doi.org/10.1145/2702123.2702190

[23] Jaeho Ryu, Naoki Hashimoto, and Makoto Sato. 2003. Analysis of Vection using Body Sway in Immersive Virtual Environment. Technical report of IEICE. Multimedia and virtual environment 103, 107 (jun 2003), 63-68. https: //ci.nii.ac.jp/naid/110003270304/en/

[24] X M Sauvan and C Bonnet. 1995. Spatiotemporal Boundaries of Linear Vection. Percept Psychophys 57, 6 (1995), 898-904. https://www.ncbi.nlm.nih.gov/pubmed/ 7651813 PMID: 7651813.

[25] Steven Strachan, Parisa Eslambolchilar, Roderick Murray-Smith, Stephen Hughes, and Sile O'Modhrain. 2005. GpsTunes: Controlling Navigation via Audio Feedback. In Proceedings of the 7th International Conference on Human Computer Interaction with Mobile Devices \&Amp; Services (MobileHCI '05). ACM, New York, NY, USA, 275-278. https://doi.org/10.1145/1085777.1085831 
[26] Satoshi Tanaka and Hiroyuki Nakano. 2019. js-STAR version 9.1.8j, Accessed on Feb 2019. http://www.kisnet.or.jp/nappa/software/star/

[27] Kenji Tanakat, Junya Hayashi, Ichiro Kawabuchi, Masahiko Inami, and Susumu Tachi. 2004. TWISTER III: A panoramic autostereo display for motion pictures. Kyokai foho Imeji Zasshi/fournal of the Institute of Image Information and Television Engineers 58, 6 (6 2004), 819-826.

[28] Koji Tsukada and Michiaki Yasumura. 2004. ActiveBelt: Belt-Type Wearable Tactile Display for Directional Navigation. In UbiComp 2004: Ubiquitous Computing, Nigel Davies, Elizabeth D. Mynatt, and Itiro Siio (Eds.). Springer Berlin Heidelberg, Berlin, Heidelberg, 384-399.

[29] Jinglong WU, Yinghua YU, and Jiajia YANG. 2013. Dependence of Luminance on the Perception of Linear Vection under Different Spatial Frequency Conditions. The fapanese fournal of Ergonomics 49, 1 (2013), 18-24. https://doi.org/10.5100/ jje. 49.18

[30] Noriko Yamagishi and Stephen J. Anderson. 1998. Localization of colour and motion stimuli in human peripheral vision. IEICE technical report. Human information processing 98, 397 (nov 1998), 23-30. https://ci.nii.ac.jp/naid/110003272306/

[31] Hiromi Yoshikawa, Taku Hachisu, Shogo Fukushima, Masahiro Furukawa, and Hiroyuki Kajimoto. 2011. "Vection Field" for Pedestrian Traffic Control. In ACM SIGGRAPH 2011 Emerging Technologies (SIGGRAPH '11). ACM, New York, NY, USA, Article 21, 1 pages. https://doi.org/10.1145/2048259.2048280

[32] Atsushi Sakuma Masahiko Inami Taro Maeda Yukata Kunita, Naoko Ogawa and Susumu Tachi. 2001. Immersive Autostereoscopic Display, Twister. The journal of the Institute of Image Information and Television Engineers 55, 5 (2001), 671-677. https://doi.org/10.3169/itej.55.671 